\title{
The Advent of Digital Productivity Assistants: The Case of Microsoft MyAnalytics
}

\author{
Michael Winikoff and Jocelyn Cranefield and Jane Li and Alexander Richter and Cathal Doyle \\ Victoria University of Wellington, New Zealand \\ \{firstname.lastname\}@vuw.ac.nz
}

\begin{abstract}
Modern digital work environments allow for great flexibility, but can also contribute to a blurring of work/life boundaries and technostress. An emerging class of intelligent tools, that we term Digital Productivity Assistant (DPA), helps knowledge workers to improve their productivity by creating awareness of their collaboration behaviour and by suggesting improvements. In this revelatory case study, we combine auto-ethnographic insights with interview data from three organisations to explore how one such tool works to influence collaboration and productivity management behaviours, using the lens of persuasive IS design. We also identify barriers to DPAs' effective use as a partner in personal productivity management.
\end{abstract}

\section{Introduction}

IT is increasingly disrupting the nature of human work. Many companies provide their employees with digital work spaces that connect them with their colleagues across countries and time zones in diverse synchronous (e.g. real-time video conferences) and asynchronous (e.g. social media) ways [1, 2, 3]. The multiplicity of communication tools gives employees greater autonomy and flexibility and allows them to stay connected with others [4]. Ultimately, they allow completely new work paradigms, like that of Digital Nomads [5].

However excessive flexibility and connectivity come with negative effects. Studies have shown that an increasing number of employees struggle with information overload or technostress [6, 7], as the increasing volume of IT-related communication often leads to reduced productivity and well-being [8].

An emerging class of intelligent tools, that we call Digital Productivity Assistants (DPAs), aims to help knowledge workers make better-informed decisions around their productivity and work-life balance. The DPAs provide overviews of their work patterns, such as collaboration behaviour, work hours, and time dedicated to focused work. Often DPAs also provide actionable advice in the form of suggestions on how individuals can change their collaboration behaviour by making them aware when they spend too much time in meetings or multi-tasking and suggesting that they plan more 'focus time' (time that is free of meetings and other interruptions) in their calendar in advance. For their analysis, they process a large amount of collaboration (meta) data (e.g. from emails, calendar appointment details) and apply a range of techniques, like comparative metrics and evidence-based justifications for the desirability of particular changes, in order to suggest modifications to work routines and nudge users towards desired behaviour. In order to provide support for behavioural modification, DPAs employ artificial intelligence techniques ("AI-powered suggestions" [9]), for example, natural language processing when analysing email content in order to identify assigned tasks.

DPAs have the potential to help users to improve their productivity by creating awareness of their collaboration behaviour and suggesting improvements in order to increase their productivity. Through acting as an analyst and adviser on productivity-related behaviours DPAs can be seen as emergent collaboration partners for knowledge workers; an application of the emergent phenomenon of machine-human collaboration [10, 11].

Given the novelty and promise of such tools, we therefore position as our first research question: How can DPAs act as partners to help improve personal productivity management?

The motivation for this study occurred with the unexpected implementation (in May 2018) of Microsoft MyAnalytics in the paper authors' workplace. We recognised this as a valuable opportunity to conduct a revelatory case study. However, we realised from our own experience that there was also a number of barriers in using the tool effectively.

We therefore ask our second research question: What 
barriers can hinder the effective use of DPAs as partners in personal productivity management?

In the following, we firstly review related studies in the context of workplace personal analytics and persuasive information systems, systems geared to promote behavioural change (Section 2). Next, we outline our methodology (Section 3) and present a case study on the use of Microsoft MyAnalytics (Section 4). In order to answer the first research question we firstly outline practices in managing productivity with MyAnalytics. Secondly, we analyse how the tool works to influence collaboration and productivity management behaviours, using the lens of persuasive IS design [12] to discuss our results. Next, we turn to the second research question and we identify barriers to effectively adopting the tool (Section 5). Finally, we conclude (Section 6) with a brief discussion of the paper's contribution, relevance to practice, and future work.

\section{Theoretical Background}

\subsection{Enterprise Personal Analytics}

Personal analytics involves using information systems to access data-based visualisations about aspects of the users' lives, with the goals of self-monitoring and self-optimisation [13] and to achieve objectives in their work and/or personal lives [14]. Catalysed by advances in mobile computing, business intelligence, and social technologies, it is still emerging as an area of academic study [14, p. 31]. Tools that support personal analytics are also known as personal informatics systems [15, 16, 17], and there is a range of tools that allow users to combine time tracking with various features to promote more efficient time management across personal and work contexts (e.g. Forest, RescueTime, and Timely).

Recently, personal analytics has entered the enterprise analytics arena, and the term enterprise personal analytics has been coined to describe this emerging group of systems which organizations use to enable workers to better manage their digital working lives [14]. Enterprise personal analytics tools share the goal of supporting reflection and guiding behavioural change, by providing information about their work patterns. However, achieving behavioural change through information is challenging: multiple barriers to use, continuance and change have been found to impact on the success of personal informatics [18]. Some of these relate to system design. For example, in a study of use of the productivity app RescueTime, Collins et al. [18] identified four barriers that inhibited user engagement and reduced the app's potential to facilitate behavioural change: data salience, the lack of contextual information, credibility of presented data and the absence of action advice. Other barriers arise from the well-documented human resistance to behavioural change, even when the target change is seen as desirable (e.g. eating more healthily). In a literature review, Kersten-van Dijk et al. [19] apply the trans-theoretical stages of change model to demonstrate that personal informatics tools are fairly successful in raising awareness of issues, and helping with behaviour maintenance, but that they provide little support for the critical early stage of change. Notably, users in the reviewed studies often found that insights were not actionable. In other words, the applications did not help them "to come up with specific strategies for tackling the problem or to build the confidence needed to translate those plans into action" [19, p. 289]. It is therefore pertinent to consider a class of tools that are more explicitly designed with the goal of changing users' attitudes and/or behaviours: Persuasive information systems.

\subsection{Persuasive Information Systems}

Persuasive information systems [12] or technologies [20] are designed to foster change in people's attitudes and/or behaviours, so as to promote the uptake of behaviours seen as beneficial to individuals and society while avoiding harmful behaviours [20, 12, 21]. This class of systems can be seen as having strong contemporary salience due to the increasing governmental interest in changing attitudes and behaviours [22]. However, unlike traditional policy tools, which use mandates, bans and incentives, nudges alter "choice architecture" [23] making it easy to accept default options [24].

The recent upsurge in the use of persuasive systems is reflected in a growing number of empirical studies. Hamari et al. [25] reviewed 95 studies and found that the most studied applications of persuasive technologies (PT) were in health/exercise $(47.4 \%)$, ecological consumption (21.1\%) and education/learning $(10.5 \%)$. Of these 95 studies, $54.7 \%$ reported fully successful outcomes and another $37.9 \%$ reported partially successful outcomes. Orji and Moffat [21] found an even higher success rate in their review of 85 studies of PT in the health and wellness domain: $75 \%$ of studies reported fully positive and $17 \%$ partially positive outcomes. $80 \%$ of these studies were of apps that used a combination of persuasive strategies, with the most common motivational strategy being tracking and monitoring. These results are interesting, since they suggest that enhancing personal analytics 


\begin{tabular}{|c|c|c|c|}
\hline Organisation & Bravo & Charlie & Delta \\
\hline Background & $\begin{array}{l}\text { University in New Zealand. } \\
\text { Teaching and research as } \\
\text { primary activities. These } \\
\text { institutional goals translate } \\
\text { into individual academic roles } \\
\text { that balance teaching and } \\
\text { research, with a weaker focus } \\
\text { on various forms of service. }\end{array}$ & $\begin{array}{l}\text { Small-to-medium technology } \\
\text { consulting company. Core } \\
\text { business is conducting } \\
\text { consulting work for a range } \\
\text { of clients. Additionally, there } \\
\text { is a range of work focused } \\
\text { on managing the company } \\
\text { and growing it, including } \\
\text { networking and developing } \\
\text { business opportunities. }\end{array}$ & $\begin{array}{l}\text { Large technology company, } \\
\text { employing } \sim 6,500 \text { people, } \\
\text { with over } 20 \text { offices in a } \\
\text { number of countries. They } \\
\text { offer a wide range of services } \\
\text { and products, including } \\
\text { data centre services, cloud } \\
\text { services, consulting, IT } \\
\text { management, and bespoke } \\
\text { software development. }\end{array}$ \\
\hline $\begin{array}{l}\text { Stakeholders } \\
\text { Interviewed }\end{array}$ & $\begin{array}{l}\text { Academics, professional staff, } \\
\text { and managers }\end{array}$ & $\begin{array}{l}\text { Partner, consultants and an IT } \\
\text { technician }\end{array}$ & $\begin{array}{l}\text { Managers, developers, and an } \\
\text { enterprise architect }\end{array}$ \\
\hline $\begin{array}{l}\text { Number of } \\
\text { Interviews }\end{array}$ & 19 & 4 & 5 \\
\hline
\end{tabular}

Table 1. Background of organisations (The names of organizations and interviewees were pseudonymized)

with persuasion techniques can improve the system's effectiveness in supporting behavioural change.

Recently, Slattery et al. [22] have focused attention on the theoretical foundations of persuasion in IS research. In a systematic literature review they identify great variation in how persuasion is conceptualised. They find that IS researchers rarely engage with literature and theory that explains the process of changing attitudes and behaviours; notably with persuasion theory. Accordingly they propose a common frame of reference for moving forward with this line of research, including defining persuasion (in the context of information systems) as something that "involves one or more agents engaged in the activity of creating, reinforcing, modifying, or extinguishing beliefs, attitudes, intentions, motivations, and/or behaviours" [22, p. 43].

\section{Research Methodology}

Owing to the emergent nature of the phenomenon being studied, we conducted a single in-depth case study [26], focusing on use of a tool, Microsoft's MyAnalytics (MMA), that had been recently implemented in three organisations. To do so, we gathered two main sets of data: (1) data about the tool itself and how it interacts with users (to investigate how it interpreted and influenced personal productivity management); and (2) in-depth qualitative data through interviews about the user's views and experiences of this tool (to understand their experiences).

The three organisations are located in New Zealand and consist of a university, a small-to-medium-sized consulting firm and a large technology company.
We interviewed ${ }^{1} 28$ people across various hierarchies and departments in the three organisations between November 2019 and February 2020 (see Table 1 for an overview). Using purposive sampling we sought participants from a range of work roles who were using, or were at least aware of, the tool; and had some autonomy in how they use their work time. This resulted in a spectrum of participants from those who used the tool regularly to those who rarely used it. The interviews - each around an hour long-had three parts. Part 1 focused on the participant's general approach to managing their time; Part 2 asked about their understanding and use of MMA; and Part 3 invited participants to open a recent report from their MMA dashboard and comment on the insights and recommendations that the tool was offering. The interviews were transcribed using Temi (a software-based audio to text transcription service) then further processed manually to rectify any errors. This resulted in 498 pages of transcripts. The transcripts were then sent to the interviewees to confirm that they were satisfied with their responses, and changes were made where requested.

In addition to the interviews, we developed a data set about the tool and its interaction with the user following an autoethnographic approach [27]. This was deemed

\footnotetext{
${ }^{1}$ We also conducted a pilot study, where we conducted 25 semi-structured interviews with staff from a New Zealand university between April and November 2019. The pilot study explored individuals' perceptions and factors relating to the use of Microsoft MyAnalytics (MMA). Staff members from three divisions of a New Zealand university were interviewed, with questions covering: the user's knowledge of the tool and the motivation for its introduction, user's experience with the tool as well as their understanding and assessment of the report produced by the tool.

${ }^{2}$ All necessary ethical approvals were obtained for this research All participants were provided with the Information Sheet and signed Consent Form to participate in the interview.
} 
necessary in order to capture the continuously evolving nature of the tool (e.g. some functionality of MMA was changed during the period of this study) and to build understanding of the tool itself: how it interacts with the user and what persuasive techniques it uses to nudge the behavioural change. Using the authors' data also reduced privacy risks to interviewees. Four researchers had access to the MMA tool, reviewing and capturing insights and suggestions provided by the tool on a weekly basis. This included a weekly digest; a dashboard; an Outlook add-in; and an inline suggestion add-in (see Section 4 for more details). They captured different types of data from the tool such as: the insights the tool was providing them with about their productivity and well-being; completing a form with the suggestions the tool was making to them; and taking screenshots of the various insights and suggestions from the tool. To enrich this data set the researchers also gathered data from other sources including official documentation about the tool, video interviews with the tool's developers; the official website and the Wayback machine which provides insights about the tool's development over time.

We then took an interpretive approach [28] in analysing the collected data as it allowed us to construct an understanding of the views and experiences of the users of the tool through their own understanding of events. This consisted of analysing the transcribed interviews via qualitative data analysis techniques [29. 30] using NVivo. Following the principles of induction, interaction, and multiple iterations, the researchers first independently generated emerging themes and then collectively discussed and refined them through several iterations until consensus was reached [31]. For additional data (e.g., documentation about the tool and captured autoethnographic data) we employed an iterative sense-making approach which consisted of analysing the data, creating representations of it, and then assessing the goodness of these representations. Thus, analysing the insights and suggestions provided by the tool helped us to build a deeper understanding of the tool, identify its persuasive strategies and patterns of interaction with users.

In answering the research questions we were mindful that the impacts of intelligent systems on work practices are likely to be both complex and subtle; and that work practices exist within a broader organizational context.

\section{The Case of Microsoft MyAnalytics}

Microsoft MyAnalytics (MMA) was introduced in $2016^{3}$ to help workers make decisions around their

$\sqrt[3]{\text { https://tinyurl.com/MMA-announce }}$ productivity and work/life balance. Based on analysis of data from the Microsoft ecosystem, it was part of a "broader vision for reinventing productivity with the intelligent cloud" [32]. With some 850 million meetings per month and billions of contacts and relationships being shared via Office 365, Microsoft had begun applying machine learning and analytics to deliver individual insights from this data, with strict privacy controls [32]. At the time of our study MMA provided analytics in the following four high-level categories.

Firstly, focus time: Time that is available 4 for uninterrupted individual work. Blocks of two hours or more are counted as focus time. The tool tracks focus time as a key metric.

Secondly, well-being: The tool tracks the number of quiet days per month as a key metric. Quiet days are days on which individuals send or read emails, attend meetings, take calls, or send chats only during work hours (by default 08:00-17:00 on weekdays).

Thirdly, network: Network contacts 5 are represented, showing how much time was spent on each contact 6 The tool also attempts to identify which contacts are important based on meetings, emails, etc., although the user can over-ride this.

And finally, collaboration: This category combines various data sources to provide feedback on the productivity of collaborative activities with key metrics on meetings, email, chat, and calls. For example, how often the user multi-tasked in a meeting (e.g. sending emails during the meeting), or how many emails were sent and received during each hour of the day.

For each of these categories MMA provided diverse insights linked with "AI-powered suggestions" [9]. Insights were analytical representations of the data, while suggestions were advice such as planning more "focus time" and calling for shorter meetings.

MMA draws on Office Graph to process data from sources including Outlook, Microsoft Teams, Skype for Business, OneDrive and SharePoint, and data from the organisational tenant level such as hierarchies. It interacts with users via four elements: a weekly digest, a dashboard; an optional Outlook toolbar add-in, and "inline suggestions" (in email and calendar). The weekly digest is placed by the system into the Outlook inbox (i.e. it is not sent through an email server) and provides a summary view of the four work patterns.

\footnotetext{
${ }^{4}$ Time that individuals block out on their calendar with no other attendees is counted as "time available to focus".

${ }^{5}$ The network consists of all internal and external contacts who were involved in a 2-way communication (email, chat, calls), or in meetings.

6"Total time is an estimation of the number of hours you spent in meetings, email, chats, and calls with that person in the last four weeks (both during and outside of your working hours)" (Source: https:// tinyurl.com/MMA-Network)
} 


\begin{tabular}{|l|l|l|}
\hline \multicolumn{1}{|c|}{ Difficulties } & \multicolumn{1}{|c|}{ Practices without MMA } & \multicolumn{1}{c|}{ Emerging Practices with MMA } \\
\hline $\begin{array}{l}\text { Juggling, } \\
\text { Interruptions, Focus, }\end{array}$ & $\begin{array}{l}\text { Plan time taking into account priorities } \\
\text { \& deadlines, (paper) to-dos, block time, } \\
\text { move meetings to create focus time, work } \\
\text { remotely to avoid disruptions }\end{array}$ & $\begin{array}{l}\text { Book focus time using MMA, share } \\
\text { calendar with colleagues so they are aware } \\
\text { of focus time bookings and can avoid } \\
\text { interrupting unless it is urgent. }\end{array}$ \\
\hline Relying on others & Plan collaboration & $\begin{array}{l}\text { Use networking tool to track who is getting } \\
\text { too little/much attention; inline suggestion } \\
\text { of outstanding tasks }\end{array}$ \\
\hline $\begin{array}{l}\text { Performing dual roles, } \\
\text { workload, institutional } \\
\text { incentives misaligned }\end{array}$ & $\begin{array}{l}\text { Work extra (nights, weekends), shorter } \\
\text { meetings (where possible!) }\end{array}$ & $\begin{array}{l}\text { Plan for more effective meetings agenda, } \\
\text { consider which ones to attend, shorter } \\
\text { meetings) - MMA does not offer much } \\
\text { help with managing dual roles }\end{array}$ \\
\hline Managing well-being & Plan time for well-being, exercise & $\begin{array}{l}\text { Track well-being, including email } \\
\text { access time patterns; prompt to turn off } \\
\text { notifications and read email less frequently }\end{array}$ \\
\hline Generic & $\begin{array}{l}\text { Review time use and self-monitoring, } \\
\text { self-moderation; Email management } \\
\text { (including inbox zero), going paperless; } \\
\text { group norms and practices }\end{array}$ & \begin{tabular}{l} 
Reflect on behaviours as seen by MMA. \\
\hline
\end{tabular} \\
\hline
\end{tabular}

Table 2. Difficulties and practices

Individuals can access a detailed MMA dashboard through a browser to drill down into insights and suggestions linked with each category. The optional Outlook add-in provides an "Insights" pane within Outlook. It provides insights based around "activity cards" such as: email read statistics (e.g. how many people have opened emails, and the average time spent reading it); tasks to follow up on (based on email content); and meeting preparation (upcoming meetings, number of acceptances, agendas and/or attachments, and an option to book preparation time beforehand). Depending on how the tool is deployed and users' operating systems, individuals can also enable dynamic AI-driven notifications for when they are reading or writing emails, and creating or receiving meeting invitation. These notifications consist of suggestions that appear in the reading pane with suggestions about how to manage email and run meetings.

\subsection{Work productivity practices}

To understand how MMA was being used, and how it could be used to help manage personal productivity, we need to firstly understand the workplace context: what do people find difficult, and what practices do they adopt to help them deal with their work.

Interviewees discussed a range of workplace difficulties that they faced in relation to productivity and well-being. The most commonly discussed issue was the need to "juggle" multiple roles and parallel activities. Other difficulties included finding time to focus, dealing with distractions and interruptions, having too much to do, having to wait for others, and misaligned institutional incentives.

In response to these difficulties, interviewees reported adopting a range of practices, some involving MMA, some not. In general, each person's practices were specific to their role and the difficulties they faced, and also reflected personal preferences (e.g. preferring to use a paper-based calendar). These practices are summarised in Table 2 and illustrated in the vignette below which is a composite, bringing together some common themes across a number of interviews.

Case vignette: Brian is a manager in Information Technology Services at a university. In his role he is responsible for a range of systems and services and manages a dozen direct reports. He is also still covering for his old role (as a systems administrator). At times it is hard for him to find time to focus, and "continually juggling priorities". Brian uses various tools to help him plan and manage his work life: calendars, to-do lists, planner tasks, project tasks, and service desk workflows. All these tools are in the Microsoft ecosystem. He plans his time on a weekly basis, using the Microsoft MyAnalytics tool to book focus time, and "then $[I]$ look around that and either duplicate that or move it around or move meetings around if I can to be able to give me some contiguous time". He shares his calendar with his team, so they can see when they are impinging on his focus time. Brian finds it useful to see who he is spending an excessive amount of time responding to: "I can see, for instance, 
I've got one project manager that I deal an awful lot with, compared to some of the other project managers. It just happens that all of that focus is on projects that he is putting through. So it's handy because I can then try and back him off slightly to be able to prioritise something else". He also finds it useful to track the extent to which he is working outside of working hours: "One of the main things I found out was... I don't have down time ... I can't remember the last time of, in MyAnalytics speak, having a quiet day. So that is where I haven't read an email or haven't done any work, outside of working hours". In response to MMA's feedback on the lack of quiet time, Brian has reflected on his behaviours, and experimented with trying to limit evening work.

\subsection{The tool as a persuasive partner}

In this section we examine how MMA collaborates with users. Specifically, we identify how it complements analytics with persuasive tactics to promote awareness of one's behaviour and suggests improvements. In doing so we draw on discourse analysis and persuasive systems design theory [12].

MMA communicates with its users via various channels that can be enabled or disabled. The nature of these communications, and user engagement with them, can be seen as critical to MMA's success. In order for users to embark on MMA's suggestions for "working smarter", MMA needs to convince users to accept its framing of their work patterns, its diagnosis of their productivity issues and its suggested solutions. It is therefore important to analyse the nature of the tool's role as an adviser and collaborator. To do this we employ the lens of communicative acts [33]. A communicative act is an act of communication that is designed to influence the receiver in some way. For example, it may involve an implicit proposal and/or a request for a co-operative response. In analysing MMA's communicative acts we also draw on Oinas-Kukkonen \& Harjumaa's persuasive design categories [12]. These authors identify four broad ways in which persuasive information systems contribute to behavioural change: these are primary task support (how the system supports the primary task), dialogue support (how the system engages with the users through dialogue), system credibility support, and social support.

MMA helps its users to increase their productivity and well-being by presenting "insights" into the last four weeks of work activity. These insights comprise short messages and graphs framed around the four categories outlined earlier: focus time, well-being, network and collaboration. The tool's use of four categories is an act of reduction: The principle of reduction is based around reducing complex behaviour into simpler tasks which can increase the benefit/cost ratio of a behaviour [12]. By omitting other ways of framing work patterns, the tool positions these four categories as those that matter most for managing productivity. The tool's use of reduction also makes it easy for users to digest and recall the information. Further, each category is linked with a persuasive rhetorical question invoking the user to review the analytics and suggestions so they can do better; e.g., Do you have enough uninterrupted time to get your work done? Could your time working with others be more productive? (These rhetorical questions imply that new activities are needed while using gain framing to make these activities seem desirable). Further, through reduction MMA positions collaboration and focus time as together comprising all work time, and as being mutually exclusive. The total time that a user spends collaborating is likely to exceed focus work time (this was the case for us) so is readily positioned as detracting from focus time. The reductive category "collaboration" thus makes it easy to view time spent collaborating as a problem. MMA's use of reduction facilitates problem framing around the need to reduce collaboration time and solution framing around the need to protect more time for focused work.

When a user seeks more detailed analytics, MMA presents a series of normative suggestions relating to performance against implicit goals. For example, in a breakdown of meeting-related behaviours it analyses performance against "[Meeting] Invitations sent with a day's notice" and "[Meeting invitations] sent with an agenda". The tool's "suggestions" combine normative framing with imperatives, such as "Respond to meetings on time". Normative framing increases the chance of user buy-in to the tool's suggestions.

The tool also employs the persuasive method of tunnelling (guiding users by providing a means for action that brings users closer to the target behaviour). For example, an opt-in tool automates the booking of daily blocks of "focus time". Offering automation of this otherwise onerous task can be seen as embedding the concept of focus time. For users who deploy this tool (done via a single click) a weekly cycle of self-monitoring of focus time is then triggered, with the tool reporting it in its weekly digest, "here's how you're doing on your plan to get daily focus time". Similarly a "shorten meeting" button encourages users of the inline insights tool to shorten a 1 hour meeting to 45 minutes in a single click. Both these examples employ the concepts of nudging and choice architecture [23]. The tool limits available choices while making a 'desirable' choice (having shorter meetings, automating the booking of focus time) readily available as low 
hanging fruit. This can also be seen as appealing to the peripheral route of change [34]: The tool automates a minor change that is in line with a larger change goal (making the monitoring of focus time a new habit). After the focus time booking tool has been enabled by a single click, MMA generates weekly reports on a user's "focus plan". This can be seen as an act of reframing in which the user's reactive deployment of the tool is recast as part of a deliberate strategy to set and monitor "your focus plan". This reframing implies an elevated level of agency and can therefore be seen as appealing to the central, goal directed system of change [34]. The tool's deployment has been mapped to a MMA-assigned productivity goal that requires ongoing monitoring and on which MMA provides feedback. The tool now prompts the user with subtle imperatives that relate to new work activities concerning time protection: Plan ahead, Needs focus time; Needs review. In combination, these communicative acts show MMA's potential to play a powerful persuasive role.

The opt-in "Inline Suggestions" feature allows the tool to engage in more dynamic, interventional communicative acts. It interrupts tasks as they are being performed by users to offer suggestions relating to the current task. For example, when starting to book an hour-long meeting the tool suggests, "Could this meeting be 45 minutes? Build in some buffer time and save attendees time". Another example occurs when the user begins replying to an email from a person categorised as 'important'. In this case the tool states (in the task window), "You may have outstanding tasks for [name]. Would you like to review now?". These dynamic interventional communicative acts are highly persuasive in nature, given the empirical knowledge of the tool and its potential to offset the risk of failing to complete outstanding tasks requested by a manager.

The above mechanisms are tightly linked with communicative acts that provide system credibility support. For example, the tool's exhortations to change are backed by empirical evidence (e.g. "you did not respond to $20 \%$ of your meeting requests last month"). This can be seen as contributing to credibility but does not support the persuasive design principle of verifiability. Users cannot verify the analysis (this was a common concern raised in interviews). The tool employs only limited social support (the fourth category of Oinas-Kukkonen \& Harjumaa [12]). According to this category users are persuaded and motivated towards change by the system's leveraging of social influence. For reasons of privacy, common social support systems (social learning, social comparison, co-operation between users and competition) are not employed by the tool.
In summary, the above analysis illustrates how MMA uses a range of persuasive methods to support and guide users towards conscious personal productivity management. The analysis also demonstrates that the tool is positioned to play a collaborative role similar to that of a performance coach. It guides users towards personal change in the form of new target work behaviours and corresponding goals, and then supports users in maintaining these behaviours.

This analysis, along with the practices highlighted in the case vignette and in Table 2, answers our first research question by discussing how DPAs can, as partners, help improve personal productivity management, with the example of MMA. In the next section we identify barriers that can hinder the effective use of DPAs as partners in personal productivity management, presenting an answer to the second research question of this paper.

\section{Barriers hindering the effective use of Digital Productivity Assistants}

Our study showed that the tool's reports led to both positive and negative reactions. Individuals who were excited were typically interested in technology and were less concerned about privacy. On the other hand, some knowledge workers were worried about the amount of data MMA could access and reflected on ways to avoid using or being monitored by the tool. More importantly, our study allowed us to identify three barriers that prevent knowledge workers from using the tool effectively. In addition to these barriers, which are specific to DPAs, it is, as always, important to ensure that the deployment of a new tool is accompanied by appropriate communication as to its function and organisational purpose, training, and support.

\section{Barrier One: Lack of understanding of how tool works}

Many users felt that their organisation could have done a better job of informing them of how MMA works, and why the tool came up with particular recommendations. MMA uses a range of concepts (e.g. 'focus time', 'quiet days'), and without having an understanding of their meaning, and how they are derived, it can be easy to misunderstand what the tool is saying ${ }^{7}$ For example, a common (but wrong!) interpretation of 'quiet time' was "days that are not too busy". This meant that when an individual saw that MMA flagged a day as being quiet, but they perceived that day as being busy, then their trust in the tool was

\footnotetext{
${ }^{7}$ In the course of our data gathering, Microsoft took steps to address this, and there is now a lot more transparency.
} 
undermined. Effective use of MMA, therefore, requires that users are supported by both their organisation and Microsoft to develop a sufficient understanding of the tool. More generally, a barrier to effective use of DPAs is lack of knowledge and understanding, which can be mitigated by careful engineering for ease-of-understanding, providing just-in-time help in the tool, and providing training prior to deployment.

\section{Barrier Two: Perceived inaccuracy of the tool}

Perceived accuracy is the extent to which the tool can be seen as having an accurate view of the interviewee's (relevant) work activities. The perceived accuracy of the tool depends on visibility and workplace practices. For instance, work done outside the Office 365 ecosystem is invisible to the tool. So if someone is working late on a report, or interacting with another online system, then MMA will not recognise that they are even working. Similarly, meetings with incomplete information (e.g., without a list of all participants 8 , ad hoc meetings, or those scheduled in different calendars will also affect correct representation of user's work activities. Perceived inaccuracy of the tool may result in users mistrusting the credibility of the data and discourage them from using the tool.

The tool's perceived (and actual) accuracy can be improved by using the calendar within the Office 365 ecosystem, and ensuring that meetings with others include the others as invited participants. Additionally, if the user has many ad hoc meetings, then it may be worth adding them to the calendar as they occur, or immediately afterwards, and we did observe some people doing this. It would also help for MMA to allow users to manually indicate the nature of an appointment (e.g. whether it is a meeting, even though it may not show other participants), and to introduce additional classifications (e.g. research/teaching/service) and types of appointments (e.g. lecture). Finally, in order to avoid private email access in the evening or weekend counting as a violation of 'quiet time', it can be desirable to have a separate account for non-work email. More generally, effective use of DPAs can be hindered by a lack of accuracy, which may be caused by a mismatch between work practices assumed by the DPA's developers, and actual individual work practices. This barrier can be mitigated by having mechanisms for transparency, allowing users to understand what the tool is using, and how, so that they can adjust their work practices, or understand which aspects of the DPA should be ignored.

\footnotetext{
${ }^{8}$ MMA only counts meetings as being collaboration when other people are invited.
}

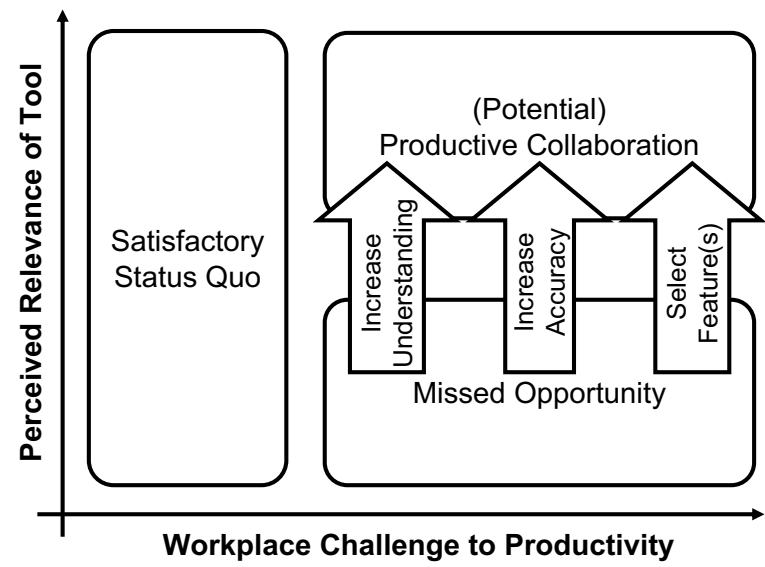

Figure 1. Approaches to overcoming the barriers

\section{Barrier Three: Lack of relevance of categories}

The categories used by MMA were frequently seen as providing a poor fit with users' work contexts. Notably the division of work activities into either focus time or collaboration did not make sense for those in certain roles. Some participants reported that they saw some meetings as an important means to achieve focused work, yet MMA designated meetings as "collaboration", implying that they detracted from "focus time". Further, some participants saw the category "focus time" as a protective container in which to place (and do) selected individual work, but others equated focus time with specific kinds of work that required them to apply intense focus (e.g. doing research). The focus time/collaboration duality also had low relevance for people whose work included activities that fitted neither category (e.g. non-collaborative meeting ${ }^{9}$, and giving lectures). Similarly, the category "quiet hours" had low relevance to those who worked long hours in evenings and weekends. More generally, for any DPA, given the wide range of work roles and activities, there is a need to understand what analytical categories are offered, and to select those features that are most relevant for a given work role. For example, for a given role insights related to focus time may be seen as irrelevant, but the analysis of how often that person checks their email may be seen as being highly relevant.

Figure 1 summarises the three responses to the barriers, placing them in context. When the user has a low level of workplace challenge that they face in their role then the status quo is satisfactory, and the tool's relevance does not matter, since there is no need for support. On the other hand, when the user faces a

\footnotetext{
${ }^{9}$ For example, a writing group, that is scheduled with multiple attendees, who turn up and use the time to write quietly.
} 
high level of challenge in their role, then a low level of relevance corresponds to a missed opportunity to benefit from DPAs. However, high challenge paired with a high level of DPA relevance corresponds to a situation where there is a (potentially) productive partnership and collaboration between the tool and the user. This relationship can aid the knowledge worker to be more productive, potentially leading to better work-life balance and better management of technostress. It is a partnership in that the human contributes by adopting particular habits that enable the DPA to have accurate information on which to base its insights and recommendations. The DPA is then able to provide relevant insights in order to change or improve digital work practices of the knowledge worker.

\section{Discussion and Conclusion}

This paper contributes to understanding an emerging class of intelligent tools that we termed "Digital Productivity Assistants", which help knowledge workers to manage their time and well-being. The tools combine attributes of Personal Analytics, providing insights about user's work and collaboration patterns as well as utilize a set of persuasive techniques to nudge changes in user's work practices. We elaborated on how DPAs act as a persuasive partner by providing actionable advice to human knowledge workers while also depending on user's adoption of practices that enable accurate representation of data and meaningful insights and recommendations. Such reciprocal relationships, if done well, leads towards productive partnerships and effective collaboration between the DPA and the user.

We provide insights into how these tools are used, showing that they do indeed have the ability to support workers in making positive changes and have emergent capabilities as teammates (collaborators in managing personal productivity). Our empirical study, thus, supports previous conceptualisation about effective collaboration between humans and AI [10, 11]. The case of MMA, as an example of DPA, demonstrated that the DPA was able to help workers identify problems affecting personal productivity, provide empirical evidence to support diagnosis, suggest actionable solutions, and facilitate uptake of these solutions through a combination of empirical evidence (analytics) and persuasive features geared at behavioural change.

However our study has also demonstrated that there can be critical barriers to knowledge workers accepting machines as teammates and particularly in accepting their problem diagnoses. We, therefore, extend previous studies on the barriers for the successful use of personal analytics tools [18], and identify three main barriers that hinder successful collaboration and effective partnership between DPAs and the user. We described these barriers along with recommended approaches to overcoming them. In order for DPAs to be effective collaborators it is necessary for them to frame information in ways that are seen as personally salient, and to be able to engage in meaningful dialogue rather than one-way communicative tactics. Notably, our analysis highlighted that a key feedback loop is missing: there is no way for the worker to provide feedback to a collaborative DPA about its interaction and about the analytical concepts (in this case relating to productivity and wellbeing) that are meaningful to a user.

In order to be able to reap the benefits of DPAs, we need to have a better understanding of how they are used in a social context, and what factors affect their use. In particular, the tool has embedded (and implicit) norms and values which are not necessarily wrong, but often do not take into account different contexts and, therefore, may be in conflict with the norms perceived by a user. This study's demonstration of the persuasive methods by DPA raises awareness of the potential of machines to act as a coach - a teammate whose focus is on assisting workers in managing personal productivity and that applies agency, in the form of persuasive techniques, to facilitate changes, subject to worker's agreement.

Our study has a number of limitations. We interviewed less than 30 people, many from a university context (although in a range of roles), and all based in New Zealand, where there is a good awareness of well-being and the need for work-life balance. Although we did interview a wide range of roles, and there were common patterns, interviewing more people, including in particular people outside New Zealand, would strengthen our conclusions. Finally, we have considered a single exemplar DPA: Microsoft MyAnalytics. Although some of our findings are more generally applicable, some are specific to MMA. Therefore, one of the next steps of our study will be to explore other DPAs and their use in other companies and cultures.

\section{References}

[1] K. Dery, I. M. Sebastian, and N. van der Meulen, "The Digital Workplace is Key to Digital Innovation," MIS Quarterly Executive, vol. 16, no. 135, pp. 135 - 152, 2017.

[2] G. C. Kane, M. Alavi, G. Labianca, and S. P. Borgatti, "What's different about social media networks? a framework and research agenda," MIS quarterly, vol. 38, no. 1, pp. 275-304, 2014.

[3] P. M. Leonardi, M. Huysman, and C. Steinfield, 
"Enterprise social media: Definition, history, and prospects for the study of social technologies in organizations," Journal of Computer-Mediated Communication, vol. 19, no. 1, pp. 1-19, 2013.

[4] M. Kügler, S. Dittes, S. Smolnik, and A. Richter, "Connect me! antecedents and impact of social connectedness in enterprise social software," Business \& Information Systems Engineering, vol. 57, no. 3, pp. 181-196, 2015.

[5] S. Richter and A. Richter, "Digital nomads," Business \& Information Systems Engineering, vol. 62, no. 1, pp. 77-81, 2020.

[6] R. Ayyagari, V. Grover, and R. Purvis, "Technostress: technological antecedents and implications," MIS Quartely, vol. 35, no. 4, pp. 831-858, 2011.

[7] M. Tarafdar, A. Gupta, and O. Turel, "The dark side of information technology use," Information Systems Journal, vol. 23, no. 3, pp. 269-275, 2013.

[8] M. Mazmanian, W. J. Orlikowski, and J. Yates, "The autonomy paradox: The implications of mobile email devices for knowledge professionals," Organization science, vol. 24, no. 5, pp. 1337-1357, 2013.

[9] K. Janardhan, "Minimize distractions and stay focused with AI-powered updates in Microsoft 365." https://tinyurl.com/minimisedistractionsJanardhan 2016. Retrieved February 29, 2020.

[10] I. Seeber, E. A. C. Bittner, R. O. Briggs, G. de Vreede, T. de Vreede, D. A. Druckenmiller, R. Maier, A. B. Merz, S. Oeste-Reiß, N. L. Randrup, G. Schwabe, and M. Söllner, "Machines as teammates: A collaboration research agenda," in 51st Hawaii International Conference on System Sciences HICSS (T. Bui, ed.), pp. 1-10, AIS Electronic Library (AISeL), 2018.

[11] I. Seeber, E. A. C. Bittner, R. O. Briggs, T. de Vreede, G. de Vreede, A. Elkins, R. Maier, A. B. Merz, S. Oeste-Reiß, N. L. Randrup, G. Schwabe, and M. Söllner, "Machines as teammates: A research agenda on AI in team collaboration," Inf. Manag., vol. 57, no. 2 , p. 103174,2020

[12] H. Oinas-Kukkonen and M. Harjumaa, "Persuasive systems design: Key issues, process model, and system features," Communications of the Association for Information Systems, vol. 24, no. 1, p. 28, 2009.

[13] M. Ruckenstein, "Visualized and interacted life: Personal analytics and engagements with data doubles," Societies, vol. 4, no. 1, pp. 68-84, 2014.

[14] T. Clohessy and T. Acton, "Enterprise personal analytics: Research perspectives and concerns," International Journal of Business Intelligence Research (IJBIR), vol. 8 , no. 2, pp. 31-48, 2017.

[15] R. Gulotta, J. Forlizzi, R. Yang, and M. W. Newman, "Fostering engagement with personal informatics systems," in Proceedings of the 2016 ACM Conference on Designing Interactive Systems (M. Foth, W. Ju, R. Schroeter, and S. Viller, eds.), pp. 286-300, 2016.

[16] S. L. Jones and R. Kelly, "Dealing with information overload in multifaceted personal informatics systems," Human-Computer Interaction, vol. 33, no. 1, pp. 1-48, 2018.

[17] I. Li, A. K. Dey, and J. Forlizzi, "A stage-based model of personal informatics systems," in Proceedings of the 28th International Conference on Human Factors in Computing Systems (E. D. Mynatt, D. Schoner, G. Fitzpatrick, S. E. Hudson, W. K. Edwards, and T. Rodden, eds.), pp. 557-566, ACM, 2010.
[18] E. I. M. Collins, A. L. Cox, J. Bird, and C. Cornish-Tresstail, "Barriers to engagement with a personal informatics productivity tool," in Proceedings of the 26th Australian Computer-Human Interaction Conference on Designing Futures: The Future of Design, p. 370-379, ACM, 2014.

[19] E. T. Kersten-van Dijk, J. H. Westerink, F. Beute, and W. A. IJsselsteijn, "Personal informatics, self-insight, and behavior change: A critical review of current literature," Human-Computer Interaction, vol. 32, no. 5-6, pp. 268-296, 2017.

[20] B. J. Fogg, "Persuasive technology: Using computers to change what we think and do," Ubiquity, Dec. 2002.

[21] R. Orji and K. Moffatt, "Persuasive technology for health and wellness: State-of-the-art and emerging trends," Health Informatics Journal, vol. 24, no. 1, pp. 66-91, 2018.

[22] P. Slattery, R. Vidgen, and P. Finnegan, "Persuasion: An analysis and common frame of reference for is research," Communications of the Association for Information Systems, vol. 46, no. 1, p. 3, 2020.

[23] R. H. Thaler and C. R. Sunstein, Nudge: Improving decisions about health, wealth, and happiness. Yale University Press, 2008.

[24] S. Benartzi, J. Beshears, K. L. Milkman, C. R. Sunstein, R. H. Thaler, M. Shankar, W. Tucker-Ray, W. J. Congdon, and S. Galing, "Should governments invest more in nudging?," Psychological Science, vol. 28, no. 8, pp. 1041-1055, 2017.

[25] J. Hamari, J. Koivisto, and T. Pakkanen, "Do persuasive technologies persuade? - a review of empirical studies," in Proceedings of the 9th International Conference on Persuasive Technology, p. 118-136, Springer-Verlag, 2014.

[26] R. K. Yin, Case study research: Design and methods. SAGE, fourth ed., 2009

[27] L. Anderson, "Analytic autoethnography," Journal of Contemporary Ethnography, vol. 35, no. 4, pp. 373-395, 2006.

[28] G. Walsham, "Doing interpretive research," European Journal of Information Systems, vol. 15, no. 3, pp. 320-330, 2006.

[29] J. T. DeCuir-Gunby, P. L. Marshall, and A. W. McCulloch, "Developing and using a codebook for the analysis of interview data: An example from a professional development research project," Field Methods, vol. 23, no. 2, pp. 136-155, 2011.

[30] M. Zachry and C. Thralls, Communicative practices in workplaces and the professions: Cultural perspectives on the regulation of discourse and organizations. Routledge, 2007.

[31] G. Walsham, "Interpretive case studies in is research: Nature and method," European Journal of Information Systems, vol. 4, no. 2, pp. 74-81, 1995.

[32] Microsoft, "Introducing MyAnalytics in Office 365." https://www.youtube.com/watch?v=43i-1Xo4wN8 2016. Retrieved February 28, 2020.

[33] W. Reich, "The cooperative nature of communicative acts," Journal of Pragmatics, vol. 43, no. 5, pp. 1349 - 1365, 2011.

[34] R. Petty and J. Cacioppo, Communication and Persuasion: Central and Peripheral Routes to Attitude Change. Springer-Verlag New York, 1986. 